\title{
Sudden death in chacma baboons (Papio ursinus)
}

\author{
C. de Villiers ${ }^{1} \& J$. V. Seier ${ }^{2}$
}

During a period of 5 months, four cases of acute death occurred in singly caged chacma baboons (Papio ursinus). Three animals were females (two adults and one young subadult) and one animal was an adult male.

The animals belonged to a colony of 90 baboons maintained at the Delft Animal Facility of the South African Medical Research Council. Animals were housed singly, outdoors (Fig. 1), with full visual, olfactory and auditory contact with conspecifics, in accordance with the South African National Guidelines for the Care and Use of Animals for Scientific Purposes. Tactile contact, including grooming with adjacent individuals, was possible through grooming panels of wire mesh. The baboons' diet consisted of $200 \mathrm{~g}$ of a pelleted feed (Aquafeeds, Cape Town, South Africa) each morning and $200 \mathrm{~g}$ of fresh fruit (apple or orange) or vegetables (sweet potato or carrot) each afternoon. Once per week, the diet was supplemented with $100 \mathrm{~g}$ of bread covered with $5 \mathrm{ml}$ of vitamin C syrup. Fresh water was supplied twice daily.

The four baboon deaths occurred within a 5-month period during 2008. All the animals were in good health on the day before they died. We had housed the

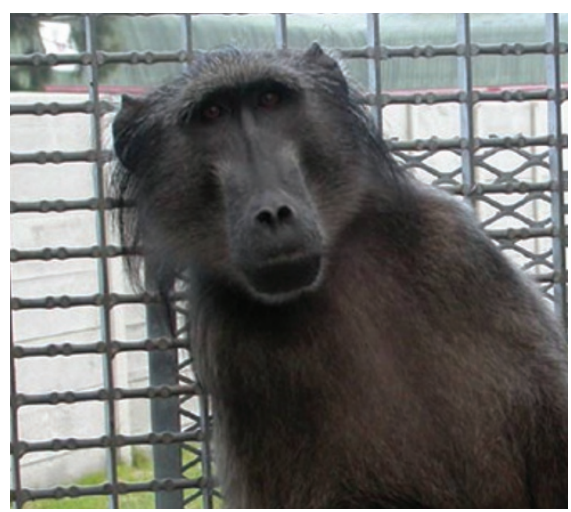

FIGURE 1 | Adult chacma baboon in outdoor housing. baboons singly and outdoors for 4 years and had not experienced overt clinical problems or unusual deaths in the colony before. Three of the baboons died at night and were found dead in their cages by caretakers during morning rounds. Caretakers found the fourth baboon in a semi-comatose state, lying in a crouched position on the cage floor. The animal's abdomen was firm and grossly distended, and this baboon died during clinical examination.

All four cases showed extreme abdominal distension. The carcasses of three baboons showed mild rectal prolapse (Fig. 2), and the prepuce was swollen on the carcass of the young subadult female (Fig. 3). The oral cavity of one animal contained ingesta.

On examination during necropsy, we noted that the carcasses of all cases were in good condition. Each animal had a dilated stomach that severely compressed the surrounding abdominal organs and diaphragm. In addition to gas, the stomach of each animal contained large amounts of water ( 3-5 l) mixed with $\sim 500 \mathrm{~g}$ of poorly digested food. Depending on what the animal had been fed the day before it died, the food in the stomach consisted of a mixture of food pellets and either sweet potato or carrots. In one animal, the stomach showed two areas of rupture that we interpreted as post-mortem lesions.

In all cases, we also saw marked subcutaneous emphysema extending to the groin, superficial muscles of the hind legs, axillae, neck and facial areas. The adult male baboon had intramuscular ecchymotic hemorrhages over the inguinal area and additional subcutaneous emphysema of the thorax. In two animals, the lungs showed marked multifocal consolidation, congestion and edema; one animal showed multifocal pulmonary ecchymotic and tracheal petechial hemorrhages. Inhaled ingesta were found in the bronchi of one animal.

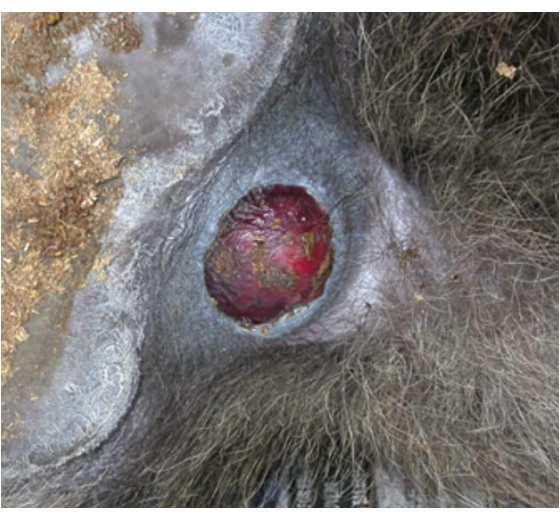

FIGURE 2 | Rectal prolapse in an adult baboon found dead in its cage.

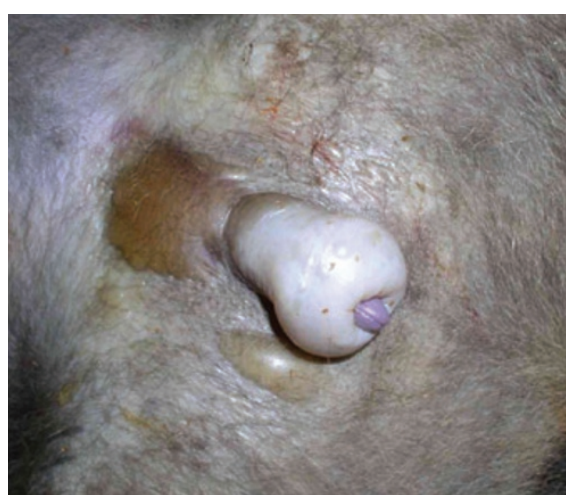

FIGURE 3 | Swollen prepuce on a young subadult female baboon found dead in its cage.

Representative tissue samples were fixed in $10 \%$ buffered formalin. After fixation, tissues were embedded in wax (Paraplast Plus, Monoject Scientific Inc.) and 4- $\mu \mathrm{m}$ sections were cut and mounted on microscopic slides for staining with hematoxylin and eosin. All sections were evaluated under a light microscope using $10 \times$ and $40 \times$ objectives.

What are your differential diagnoses for this condition? Is this condition treatable? If so, how would you treat it?

What's your diagnosis? 